\title{
Vitamin D status and cardiometabolic assessment in infancy
}

\author{
Simone Kew ${ }^{1}$, Jill K. Hamilton², Chang Ye ${ }^{3}$, Anthony J. Hanley ${ }^{1,3,4}$, Bernard Zinman ${ }^{1,3,5}$ and Ravi Retnakaran 1,3,5
}

\begin{abstract}
BACKGROUND: Infants are at risk of vitamin D insufficiency, owing to their limited exposure to direct sunlight and the low levels of vitamin D in breast milk. Although vitamin D insufficiency has been associated with cardiometabolic risk factors in children, these associations have not been studied in infants, despite their unique risks. Therefore, we sought to determine whether vitamin D status was associated with cardiometabolic measures in infants.
\end{abstract}

METHODS: Ninety-nine full-term infants were evaluated at the age of 1 y with measurement of 25-hydroxy vitamin D (25-OH-D) and an array of traditional (fasting glucose, insulin, low-densitylipoprotein cholesterol, high-density-lipoprotein cholesterol, triglycerides) and emerging (C-reactive protein, adiponectin, leptin) cardiometabolic risk factors. On the basis of 25-OH-D levels, infants were classified as vitamin D sufficient $(n=59)$, vitamin D insufficient ( $n=29)$, or vitamin D deficient $(n=11)$.

RESULTS: Duration of exclusive breastfeeding and prevalence of nonwhite ethnicity were highest in the vitamin D-deficient group ( $P=0.05$ and 0.03 , respectively). Current use of vitamin $D$ supplementation was highest in the sufficient group $(P=0.02)$. Of note, however, there were no significant differences among the three groups in any of the cardiometabolic risk factors, on both unadjusted and covariate-adjusted analyses.

CONCLUSION: Vitamin D insufficiency/deficiency is not associated with an adverse cardiometabolic risk factor profile in 1 -y-old infants.

$\mathbf{T}$ he World Health Organization recommends exclusive breastfeeding for the first 6 mo of life, followed by continued breastfeeding coupled with complementary foods for up to 2 y (1). However, because human breast milk has low levels of vitamin $\mathrm{D}$, it is recognized that exclusively breastfed infants can be at risk for deficiency of this essential vitamin (2). Moreover, because cutaneous synthesis in response to sunlight is the main source of vitamin $\mathrm{D}$ in humans, infants are at further risk of vitamin D insufficiency because excessive sun exposure is not recommended under the age of $1 \mathrm{y}$ (3). This risk may be particularly enhanced in children of nonwhite ethnicity, in whom melanin can interfere with sunlight-mediated cutaneous synthesis (4). Therefore, in light of these considerations, it is recommended that breastfed infants receive dietary supplementation of vitamin D (5).
The clinical basis for infant vitamin D supplementation has traditionally focused on support for the normal growth and development of the skeletal system. Besides its classical role in calcium and bone homeostasis, however, vitamin $\mathrm{D}$ is now known to affect many other physiologic processes, including vascular function and metabolism (6,7). Indeed, in adults, a growing body of evidence has linked vitamin D insufficiency with vascular risk factors and cardiovascular disease (6). Furthermore, even in children aged 6-19 y, vitamin D insufficiency has been associated with cardiometabolic risk factors such as dyslipidemia, hypertension, and low levels of adiponectin (8-12). In this context, however, there has been little study of the cardiometabolic risk factor implications of vitamin $\mathrm{D}$ status during infancy, despite it being a time that carries unique risks for vitamin $\mathrm{D}$ insufficiency. The importance of this question is further underscored by the fact that vascular risk factors that arise in childhood are known to track into adulthood and to contribute to atherosclerotic disease (13-15). Therefore, our objective in this study was to evaluate the cardiometabolic risk factor associations of vitamin D status in infants.

\section{RESULTS}

Table 1 shows the comparison of the infants comprising the vitamin D-sufficient $(n=59)$, vitamin D-insufficient $(n=29)$, and vitamin $\mathrm{D}$-deficient $(n=11)$ groups. As expected, both duration of exclusive breastfeeding and prevalence of nonwhite ethnicity were highest in the vitamin D-deficient group (overall $P=0.05$ and 0.03 , respectively). Similarly, the prevalence of current use of infant vitamin D supplementation was highest in the sufficient group $(P=0.02)$. Otherwise, there were no significant differences between the groups in infant age, gender, maternal antepartum glucose tolerance status, birth weight, infant anthropometry at $1 \mathrm{y}$ (weight, length, BMI $Z$ score, sum of skinfolds), age of introduction of solid food, season of assessment, or maternal physical activity. Of note, there were also no differences between the three groups in any of the traditional (fasting glucose, fasting insulin, homeostasis model of assessment of insulin resistance, total cholesterol, low-densitylipoprotein, high-density-lipoprotein, total:high-density-lipoprotein cholesterol ratio, triglycerides) and emerging (leptin, adiponectin, C-reactive protein) risk factors. On Spearman 


\section{Articles | Kewet al.}

Table 1. Comparison of clinical characteristics and cardiometabolic risk factors among (i) vitamin D-deficient infants, (ii) vitamin D-insufficient infants, and (iii) vitamin D-sufficient infants

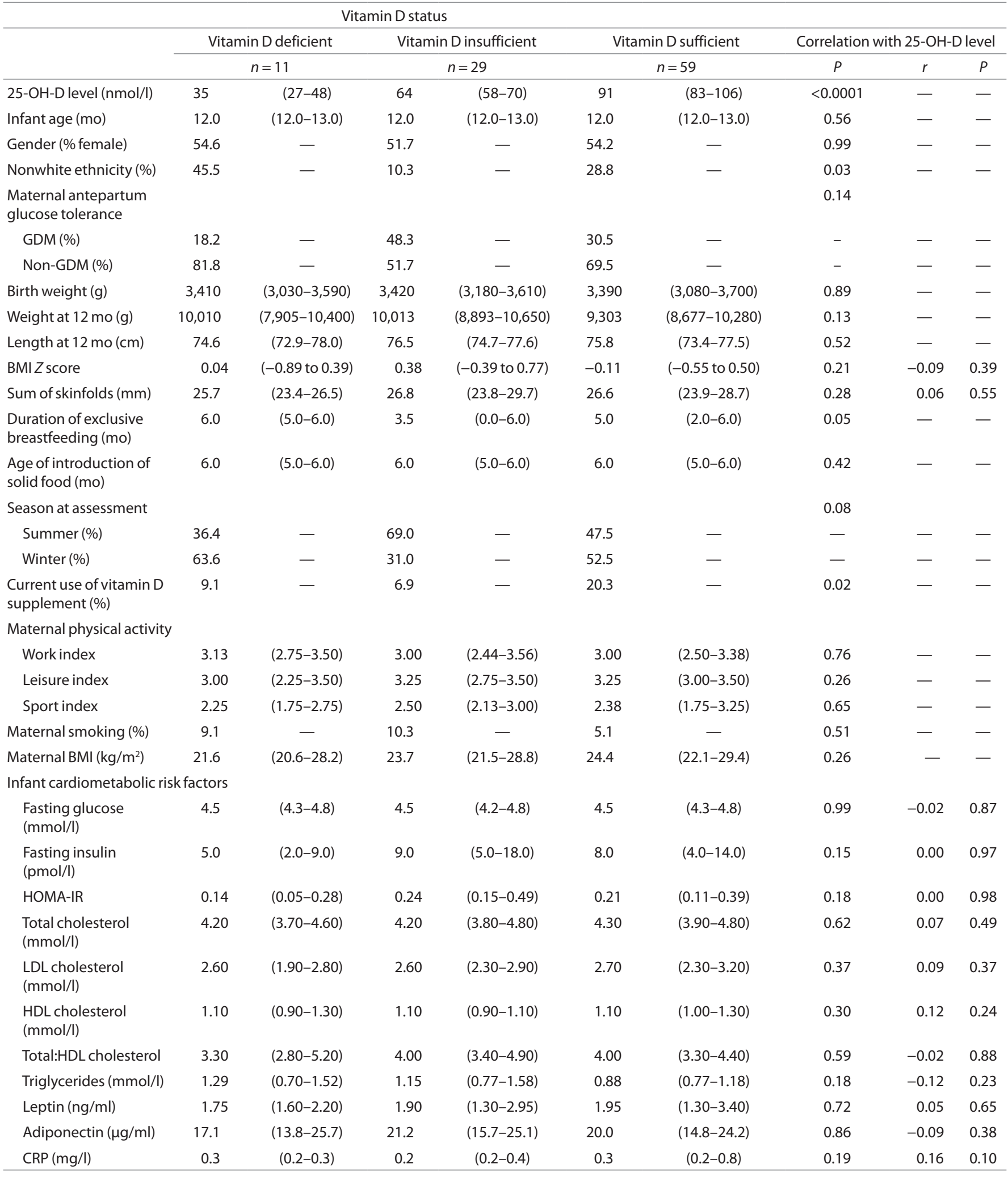

Spearman correlations of cardiometabolic risk factors with 25-hydroxy vitamin D concentrations are shown after adjustment for infant age. Continuous data are presented as median followed by interquartile range in parentheses. Categorical data are presented as percentages. $P$ values refer to overall differences across the groups as determined by the Kruskal-Wallis test for continuous variables or $\chi^{2}$ test or Fisher's exact test for categorical data. Spearman correlations are adjusted for infant age, with the exception of BMI Z score (because BMI Z score includes age).

25-OH-D, 25-hydroxy vitamin D; CRP, C-reactive protein; GDM, gestational diabetes mellitus; HDL, high-density lipoprotein; HOMA-IR, homeostasis model of assessment of insulin resistance; LDL, low-density lipoprotein. 
correlation analysis adjusted for infant age (Table 1), there were no significant correlations between 25-hydroxy vitamin $\mathrm{D}$ (25-OH-D) and any of the cardiometabolic risk factors (with $r$ values $\sim 0$ ). Furthermore, after adjustment for age, gender,

Table 2. Adjusted mean levels of cardiometabolic risk factors in infant vitamin D groups and analogous bootstrap-adjusted mean levels, after adjustment for infant age, sex, ethnicity, duration of exclusive breastfeeding, season, and sum of skinfolds

\begin{tabular}{|c|c|c|c|c|}
\hline & $\begin{array}{l}\text { Vitamin D } \\
\text { deficient }\end{array}$ & $\begin{array}{l}\text { Vitamin D } \\
\text { insufficient }\end{array}$ & $\begin{array}{l}\text { Vitamin D } \\
\text { sufficient }\end{array}$ & $P$ \\
\hline \multicolumn{5}{|c|}{ Actual-adjusted means } \\
\hline $\begin{array}{l}\text { Fasting glucose } \\
(\mathrm{mmol} / \mathrm{l})\end{array}$ & $4.5(0.19)$ & $4.5(0.13)$ & $4.6(0.09)$ & 0.83 \\
\hline $\begin{array}{l}\text { Fasting insulin } \\
(\mathrm{pmol} / \mathrm{l})\end{array}$ & $4.9(1.62)$ & $8.3(1.92)$ & $6.6(1.05)$ & 0.42 \\
\hline HOMA-IR & $0.14(0.05)$ & $0.23(0.06)$ & $0.18(0.03)$ & 0.48 \\
\hline $\begin{array}{l}\text { Total cholesterol } \\
(\mathrm{mmol} / \mathrm{l})\end{array}$ & $4.16(0.23)$ & $4.19(0.16)$ & $4.38(0.11)$ & 0.46 \\
\hline $\begin{array}{l}\text { LDL cholesterol } \\
(\mathrm{mmol} / \mathrm{l})\end{array}$ & $2.47(0.21)$ & $2.56(0.15)$ & $2.75(0.10)$ & 0.30 \\
\hline $\begin{array}{l}\mathrm{HDL} \text { cholesterol } \\
(\mathrm{mmol} / \mathrm{l})\end{array}$ & $1.13(0.08)$ & $1.10(0.05)$ & $1.17(0.04)$ & 0.50 \\
\hline $\begin{array}{l}\text { Total:HDL } \\
\text { cholesterol }\end{array}$ & $3.9(0.35)$ & $4.08(0.24)$ & $3.87(0.16)$ & 0.73 \\
\hline $\begin{array}{l}\text { Triglycerides } \\
(\mathrm{mmol} / \mathrm{l})\end{array}$ & $1.22(0.18)$ & $1.17(0.12)$ & $1.02(0.08)$ & 0.39 \\
\hline Leptin (ng/ml) & $1.93(0.38)$ & $2.21(0.30)$ & $2.10(0.20)$ & 0.85 \\
\hline $\begin{array}{l}\text { Adiponectin } \\
(\mu \mathrm{g} / \mathrm{ml})\end{array}$ & $21.6(2.10)$ & $21.7(1.40)$ & $20.4(1.00)$ & 0.68 \\
\hline $\mathrm{CRP}(\mathrm{mg} / \mathrm{l})$ & $0.4(0.10)$ & $0.3(0.10)$ & $0.4(0.10)$ & 0.17 \\
\hline \multicolumn{5}{|c|}{ Bootstrap-adjusted means } \\
\hline $\begin{array}{l}\text { Fasting glucose } \\
(\mathrm{mmol} / \mathrm{l})\end{array}$ & $4.5(0.19)$ & $4.5(0.13)$ & $4.6(0.10)$ & 0.81 \\
\hline $\begin{array}{l}\text { Fasting insulin } \\
(\mathrm{pmol} / \mathrm{l})\end{array}$ & $5.0(1.63)$ & $8.4(1.51)$ & $6.6(1.06)$ & 0.70 \\
\hline HOMA-IR & $0.14(0.05)$ & $0.23(0.04)$ & $0.18(0.03)$ & 0.72 \\
\hline $\begin{array}{l}\text { Total cholesterol } \\
(\mathrm{mmol} / \mathrm{l})\end{array}$ & $4.16(0.23)$ & $4.18(0.15)$ & $4.37(0.10)$ & 0.64 \\
\hline $\begin{array}{l}\text { LDL cholesterol } \\
(\mathrm{mmol} / \mathrm{l})\end{array}$ & $2.47(0.22)$ & $2.55(0.15)$ & $2.75(0.10)$ & 0.62 \\
\hline $\begin{array}{l}\text { HDL cholesterol } \\
(\mathrm{mmol} / \mathrm{l})\end{array}$ & $1.13(0.08)$ & $1.10(0.05)$ & $1.17(0.04)$ & 0.68 \\
\hline $\begin{array}{l}\text { Total:HDL } \\
\text { cholesterol }\end{array}$ & $3.90(0.35)$ & $4.06(0.24)$ & $3.87(0.16)$ & 0.80 \\
\hline $\begin{array}{l}\text { Triglycerides } \\
(\mathrm{mmol} / \mathrm{l})\end{array}$ & $1.21(0.18)$ & $1.16(0.12)$ & $1.02(0.08)$ & 0.69 \\
\hline Leptin (ng/ml) & $1.95(0.39)$ & $2.23(0.30)$ & $2.10(0.20)$ & 0.85 \\
\hline $\begin{array}{l}\text { Adiponectin } \\
(\mu \mathrm{g} / \mathrm{ml})\end{array}$ & $21.7(2.10)$ & $21.7(1.41)$ & $20.4(1.00)$ & 0.82 \\
\hline CRP (mg/l) & $0.4(0.11)$ & $0.3(0.05)$ & $0.4(0.05)$ & 0.63 \\
\hline
\end{tabular}

Data are presented as adjusted mean for each group, followed by SE in parentheses. $P$ values refer to overall difference between groups as determined by analysis of covariance.

CRP, C-reactive protein; HDL, high-density lipoprotein; HOMA-IR, homeostasis model of assessment of insulin resistance; LDL, low-density lipoprotein. ethnicity, duration of exclusive breastfeeding, season, and sum of skinfolds, there were still no significant differences among the three vitamin D groups in the mean-adjusted levels of any of the traditional and emerging cardiometabolic risk factors (Table 2).

To address the possibility that the sample size could have limited the capacity for detecting differences between the groups, the adjusted analyses were repeated with 2,000 bootstrap samples drawn from the original dataset with replacement. As shown in Table 2, the adjusted mean levels from the bootstrap samples were very similar to those from the original data. The bootstrap $P$ values were all nonsignificant, supporting the conclusion that the cardiometabolic risk factors were not significantly different among the three infant vitamin D groups.

Further sensitivity analyses were performed by comparing the vitamin $\mathrm{D}$-sufficient infants $(n=59)$ with those who were vitamin $\mathrm{D}$ insufficient or deficient combined $(n=40)$. As shown in Table 3, there were no differences between these two groups in adjusted mean levels of any of the cardiometabolic risk factors. Bootstrap analyses again supported this conclusion, as evidenced by the bootstrap 95\% confidence intervals for the adjusted mean differences between the two groups and the associated $P$ values (Table 3 ).

Finally, we performed multiple linear regression analysis to identify the determinants of 25-OH-D levels in the infants. This analysis revealed that after adjustment for infant age, gender, ethnicity, duration of exclusive breastfeeding, season of assessment, and sum of skinfolds, the sole independent predictor of 25-OH-D was the current use of vitamin D supplementation $(\beta=23.7, t=3.08, P=0.0027)$.

\section{DISCUSSION}

Several recent studies have found low vitamin D levels to be associated with cardiometabolic risk factors in adolescents as well as in children as young as $6 \mathrm{y}$ of age (8-12). In this context, the key finding of this study is that vitamin D insufficiency/ deficiency was not associated with any of the traditional or emerging risk factors assessed at the age of $1 \mathrm{y}$. Furthermore, because the offspring of women with gestational diabetes mellitus (GDM) have an increased risk for the early accrual of cardiovascular risk factors as compared with their peers $(16,17)$, this lack of association was observed in a cohort that reflects a spectrum of risk for future cardiometabolic disease.

The clinical relevance of these data rests in the finding that, although the recommendation of exclusive breastfeeding can potentially increase the risk of infant vitamin D insufficiency/ deficiency, the latter was not associated with an adverse vascular risk factor profile. This reassuring finding is consistent with previous data linking exclusive breastfeeding with better cardiometabolic function in adult life (18). Of note, although the sample size in the current study was modest $(n=99)$, it is unlikely that the absence of differences in risk factors between vitamin D groups was due to insufficient statistical power for three reasons. First, this sample size was sufficient to show the expected associations of vitamin $\mathrm{D}$ status with duration of exclusive 
Table 3. Adjusted mean levels of cardiometabolic risk factors in vitamin D-deficient/insufficient and-sufficient groups and analogous bootstrap-adjusted means, after adjustment for infant age, sex, ethnicity, duration of breastfeeding, season, and sum of skinfolds

\begin{tabular}{|c|c|c|c|c|}
\hline & $\begin{array}{l}\text { Vitamin D } \\
\text { deficient or } \\
\text { insufficient }\end{array}$ & $\begin{array}{l}\text { Vitamin D } \\
\text { sufficient }\end{array}$ & \multirow{2}{*}{$\begin{array}{l}95 \% \mathrm{Cl} \text { for } \\
\text { adjusted mean } \\
\text { difference }\end{array}$} & \multirow[b]{2}{*}{$P$} \\
\hline & $n=40$ & $n=59$ & & \\
\hline \multicolumn{5}{|c|}{ Actual-adjusted means } \\
\hline $\begin{array}{l}\text { Fasting glucose } \\
(\mathrm{mmol} / \mathrm{l})\end{array}$ & $4.48(0.10)$ & $4.56(0.09)$ & $(-0.32,0.17)$ & 0.55 \\
\hline $\begin{array}{l}\text { Fasting insulin } \\
(\mathrm{pmol} / \mathrm{l})\end{array}$ & $6.96(1.32)$ & $6.49(1.04)$ & $(-3.07,0.52)^{\mathrm{a}}$ & 0.75 \\
\hline HOMA-IR & $0.19(0.04)$ & $0.18(0.03)$ & $(-0.41,0.53)^{\mathrm{a}}$ & 0.80 \\
\hline $\begin{array}{l}\text { Total cholesterol } \\
(\mathrm{mmol} / \mathrm{l})\end{array}$ & $4.18(0.13)$ & $4.37(0.11)$ & $(-0.49,0.11)$ & 0.21 \\
\hline $\begin{array}{l}\text { LDL cholesterol } \\
(\mathrm{mmol} / \mathrm{l})\end{array}$ & $2.53(0.12)$ & $2.75(0.10)$ & $(-0.50,0.07)$ & 0.13 \\
\hline $\begin{array}{l}\text { HDL cholesterol } \\
(\mathrm{mmol} / \mathrm{l})\end{array}$ & $1.11(1.02)$ & $1.17(0.04)$ & $(-0.16,0.04)$ & 0.26 \\
\hline $\begin{array}{l}\text { Total:HDL } \\
\text { cholesterol }\end{array}$ & $4.02(0.19)$ & $3.87(0.16)$ & $(-0.31,0.62)$ & 0.50 \\
\hline $\begin{array}{l}\text { Triglycerides } \\
(\mathrm{mmol} / \mathrm{l})\end{array}$ & $1.18(0.10)$ & $1.02(0.08)$ & $(-0.07,0.40)$ & 0.17 \\
\hline Leptin (ng/ml) & $2.12(0.23)$ & $2.08(0.21)$ & $(-0.25,0.27)^{\mathrm{a}}$ & 0.93 \\
\hline $\begin{array}{l}\text { Adiponectin } \\
(\mu \mathrm{g} / \mathrm{ml})\end{array}$ & $21.67(1.15)$ & $20.44(1.00)$ & $(-1.53,3.99)$ & 0.38 \\
\hline $\mathrm{CRP}(\mathrm{mg} / \mathrm{l})$ & $0.29(0.05)$ & $0.38(0.05)$ & $(-0.65,0.08)^{a}$ & 0.12 \\
\hline \multicolumn{5}{|c|}{ Bootstrap-adjusted means } \\
\hline $\begin{array}{l}\text { Fasting glucose } \\
(\mathrm{mmol} / \mathrm{l})\end{array}$ & $4.48(0.10)$ & $4.56(0.08)$ & $(-0.31,0.16)$ & 0.56 \\
\hline $\begin{array}{l}\text { Fasting insulin } \\
(\mathrm{pmol} / \mathrm{l})\end{array}$ & $7.03(1.27)$ & $6.49(0.97)$ & $(-0.34,0.51)^{\mathrm{a}}$ & 0.68 \\
\hline HOMA-IR & $0.19(0.04)$ & $0.18(0.03)$ & $(-0.38,0.53)^{\mathrm{a}}$ & 0.79 \\
\hline $\begin{array}{l}\text { Total cholesterol } \\
(\mathrm{mmol} / \mathrm{l})\end{array}$ & $4.18(0.12)$ & $4.37(0.10)$ & $(-0.50,0.11)$ & 0.50 \\
\hline $\begin{array}{l}\text { LDL cholesterol } \\
(\mathrm{mmol} / \mathrm{l})\end{array}$ & $2.53(0.12)$ & $2.75(0.10)$ & $(-0.51,0.07)$ & 0.50 \\
\hline $\begin{array}{l}\text { HDL cholesterol } \\
(\mathrm{mmol} / \mathrm{l})\end{array}$ & $1.11(0.04)$ & $1.17(0.04)$ & $(-0.16,0.04)$ & 0.52 \\
\hline $\begin{array}{l}\text { Total:HDL } \\
\text { cholesterol }\end{array}$ & $4.01(0.19)$ & $3.86(0.16)$ & $(-0.32,0.63)$ & 0.58 \\
\hline $\begin{array}{l}\text { Triglycerides } \\
(\mathrm{mmol} / \mathrm{l})\end{array}$ & $1.18(0.10)$ & $1.02(0.08)$ & $(-0.07,0.39)$ & 0.54 \\
\hline Leptin (ng/ml) & $2.12(0.23)$ & $2.08(0.21)$ & $(-0.23,0.26)^{\mathrm{a}}$ & 0.93 \\
\hline $\begin{array}{l}\text { Adiponectin } \\
(\mu \mathrm{g} / \mathrm{ml})\end{array}$ & $21.67(1.14)$ & $20.37(1.00)$ & $(-1.59,4.20)$ & 0.55 \\
\hline CRP (mg/l) & $0.29(0.05)$ & $0.38(0.05)$ & $(-0.65,0.07)^{\mathrm{a}}$ & 0.49 \\
\hline
\end{tabular}

Data are presented as adjusted mean for each group, followed by SE in parentheses.

Cl, confidence interval; CRP, C-reactive protein; GDM, gestational diabetes mellitus; $\mathrm{HDL}$, high-density lipoprotein; HOMA-IR, homeostasis model of assessment of insulin resistance; LDL, low-density lipoprotein.

aFasting insulin, HOMA-IR, CRP, and leptin had skewed distributions, so natural log transformations were taken. Their confidence intervals are presented based on the natural $\log$ scale. breastfeeding, nonwhite ethnicity, and use of supplementation. Second, not only were the univariate correlations of $25-\mathrm{OH}-\mathrm{D}$ levels with all of the cardiometabolic factors nonsignificant, but they also had correlation coefficients ( $r$ values) near 0 with large $P$ values, thereby suggesting no association whatsoever. Finally, we confirmed our results with bootstrap analyses, which showed no significant differences between the vitamin $\mathrm{D}$ groups in the adjusted mean levels of any of the risk factors. Moreover, there were also no differences comparing the vitamin D-sufficient infants with those infants who were insufficient or deficient (as combined in Table 3). Therefore, taking all of these features into consideration, the current findings appear to be quite robust.

Although we found no cross-sectional associations during infancy, it remains possible that cardiometabolic risk factors may develop over a time frame beyond that of this study. Specifically, we cannot rule out the possibility that infants with vitamin $D$ insufficiency or deficiency may go on to acquire cardiometabolic risk factors during childhood as they grow older. The importance of addressing this issue with further longitudinal study is underscored by the fact that vitamin D insufficiency/deficiency is readily treatable with supplementation.

Another limitation of this study is that we did not measure levels of parathyroid hormone, calcium, phosphate, or alkaline phosphatase. In addition, although we measured the usage of vitamin $D$ supplementation at the age of $1 \mathrm{y}$, we did not determine if those not using this therapy at that time were using it when exclusively breastfeeding. As such, despite the low rates of supplementation noted in this study, it is not known whether infants had received this recommended therapy at the time when they were probably at greatest risk of insufficiency/deficiency (when exclusively breastfed). Nevertheless, we have demonstrated that the use of vitamin $\mathrm{D}$ supplementation was the sole independent predictor of infant 25-OH-D levels at the age of $1 \mathrm{y}$, after considering risk factors for insufficiency/deficiency. Furthermore, it should be recognized that the focus of this analysis was on the cardiometabolic risk factor associations of vitamin D status, as reflected by circulating levels of 25-OH-D (which are downstream of the sources of vitamin $\mathrm{D}$ ). In this regard, to our knowledge, the current study is the first to address this important question in infants and should lead to further studies.

In summary, vitamin D insufficiency/deficiency was not associated with traditional or emerging cardiometabolic risk factors in infants aged $1 \mathrm{y}$. These findings are reassuring because key infant care recommendations regarding breastfeeding and sun exposure can potentially increase the risk of vitamin D insufficiency and deficiency (if sufficient supplementation is not provided). Ultimately, further longitudinal study is required to delineate the timing whereupon associations between vitamin $\mathrm{D}$ status and cardiometabolic risk factors may first develop in childhood.

\section{METHODS}

\section{Study Population}

This analysis was conducted in the setting of an ongoing prospective observational study in which the infants from a cohort of women recruited at the time of antepartum screening for GDM are undergoing assessment in the first year of life. The study protocol has been 
previously described in detail $(19,20)$. In brief, pregnant women at our institution are screened for GDM by a $50 \mathrm{~g}$ glucose challenge test (GCT) in the late second trimester, followed by referral for a diagnostic 3-h $100 \mathrm{~g}$ oral glucose tolerance test if the GCT screen is abnormal. In this study, women are recruited either before or after their GCT, and all participants undergo a 3-h $100 \mathrm{~g}$ oral glucose tolerance test for determination of GDM status (regardless of the GCT result). The strategy of recruiting women following an abnormal GCT served to enrich the proportion of women with GDM within the study population. Women diagnosed with GDM (by National Diabetes Data Group criteria on the oral glucose tolerance test) (21) are referred to a specialized clinic for diabetes in pregnancy, where they receive glucose-lowering therapy (dietary/exercise counseling \pm insulin) in order to reduce the risk of hyperglycemia-mediated fetal overgrowth. The offspring are evaluated at delivery and in the first year of life. The study protocol has been approved by the institutional research ethics boards, and all mothers provided written informed consent for infant participation. The current analysis was restricted to the first 99 singleton, full-term infants who had completed blood work for assessment of vitamin D status at the age of $1 \mathrm{y}$ as of July 2011.

\section{Infant Assessment and Biochemical Analyses}

At the age of $1 \mathrm{y}$, infants attended the clinical investigation unit in the early morning, after a requested overnight fast (or at least $4-5 \mathrm{~h}$, if they required feeding). Mothers completed interviewer-administered questionnaires, and infant length, weight, and skinfold thickness were measured, as previously described (19). Maternal physical activity in the preceding year was assessed using the Baecke Questionnaire $(22,23)$. Fasting venous blood samples were drawn from the infants for the measurement of 25-OH-D levels and both traditional (fasting glucose, insulin, lipids) and emerging (leptin, adiponectin, C-reactive protein) cardiometabolic risk factors. 25-OH-D was measured by chemiluminescent immunoassay (Diasorin, Saluggia, Italy). Specific insulin was measured by electrochemiluminescence immunoassay kit and Elecsys 1010 immunoassay analyzer (Roche, Laval, Canada). Total cholesterol, high-density-lipoprotein cholesterol, and triglycerides were measured by enzymatic calorimetric assays (Roche, Indianapolis, IN). Low-density-lipoprotein cholesterol was determined by the Friedewald formula. Leptin was measured by enzyme-linked immunosorbent assay (Millipore, Linco, St Charles, $\mathrm{MO}$ ). Adiponectin was measured by radioimmunoassay (Millipore, Billerica, MA). C-reactive protein was measured by particle-enhanced immunoturbidimetric assay (Roche). Homeostasis model of assessment of insulin resistance was determined as described by Matthews et al. (24).

The measurement of 25-OH-D levels enabled stratification of the study population into the following three groups based on infant vitamin D status: (i) vitamin D deficient (defined as $25-\mathrm{OH}-\mathrm{D}<50$ $\mathrm{nmol} / \mathrm{l})$, (ii) vitamin D insufficient $(50 \mathrm{nmol} / \mathrm{l} \leq 25-\mathrm{OH}-\mathrm{D}<75 \mathrm{nmol} / \mathrm{l})$, and (iii) vitamin $\mathrm{D}$ sufficient $(25-\mathrm{OH}-\mathrm{D} \geq 75 \mathrm{nmol} / \mathrm{l})$. In the absence of infant-specific consensus guidelines, these 25-OH-D thresholds for defining infant vitamin D status have been applied as suggested by previous investigators and the Endocrine Society Clinical Practice Guidelines $(3,25,26)$.

\section{Statistical Analyses}

All analyses were performed using Statistical Analysis System 9.2 software (SAS Institute, Cary, NC). Continuous variables were tested for normality of distribution, with natural log transformations of skewed variables used for subsequent analysis. In Table 1, unadjusted comparisons between the three vitamin D groups were performed by Kruskal-Wallis test (for continuous variables) and $\chi^{2}$ or Fisher's exact tests (for categorical variables). Univariate associations between 25-OH-D levels and infant cardiometabolic risk factors were assessed by Spearman correlation analysis, adjusted for infant age. Mean-adjusted risk factors were compared between groups by analysis of covariance, after adjustment for age, gender, sum of skinfolds, and risk factors for vitamin D deficiency/insufficiency (ethnicity, duration of exclusive breastfeeding, and season of assessment) (Table 2).
To address the possibility that the sample size might have limited the capacity for detecting differences between the groups, we repeated this analysis in Table 2 using the bootstrap method (27). Specifically, we drew 2,000 bootstrap samples from the original data set with replacement and fitted the same model (conducting analysis of covariance) to the bootstrap samples. For each infant cardiometabolic risk factor, adjusted mean levels for the three groups (and their associated $F$ values) were obtained, enabling comparison of the adjusted means from each bootstrap sample. The bootstrap $P$ value for testing the between-group difference for each risk factor was estimated by the proportion of bootstrap $F$ values greater than or equal to the observed $F$ value from the original data.

Sensitivity analyses were performed comparing the vitamin D-sufficient infants with those who were either vitamin $D$ insufficient or deficient (i.e., combined). Adjusted mean levels of infant risk factors were compared between these two groups with bootstrap analyses repeated for these comparisons (Table 3 ).

\section{ACKNOWLEDGMENT}

We thank Mount Sinai Hospital Department of Pathology and Laboratory Medicine and Patient Care Services.

\section{STATEMENT OF FINANCIAL SUPPORT}

This study was funded by operating grants from the Canadian Institutes of Health Research (MOP-89831 and MOP-84206). R.R. holds an Ontario Ministry of Research and Innovation Early Researcher Award.

Disclosure: The authors declare no conflict of interests.

\section{REFERENCES}

1. World Health Organization. Innocenti declaration on the protection, promotion and support of breastfeeding. Geneva, Switzerland: WHO, 1991. (http://www.unicef.org/programme/breastfeeding/innocenti.htm).

2. Institute of Medicine Food and Nutrition Board Standing Committee on the Scientific Evaluation of Dietary Reference Intakes. Dietary reference intakes for calcium, phosphorous, magnesium, vitamin D, and fluoride. Washington, DC: National Academy Press, 1997:263-85.

3. Wagner CL, Greer FR; American Academy of Pediatrics Section on Breastfeeding; American Academy of Pediatrics Committee on Nutrition. Prevention of rickets and vitamin $\mathrm{D}$ deficiency in infants, children, and adolescents. Pediatrics 2008;122:1142-52.

4. Hanley DA, Davison KS. Vitamin D insufficiency in North America. J Nutr 2005;135:332-7.

5. American Academy of Pediatrics Section on Breastfeeding. Breastfeeding and the use of human milk. Pediatrics 2005;115:496-506.

6. McGreevy C, Williams D. New insights about vitamin D and cardiovascular disease: a narrative review. Ann Intern Med 2011;155:820-6.

7. Stivelman E, Retnakaran R. Role of vitamin D in the pathophysiology and treatment of type 2 diabetes. Curr Diabetes Rev 2012;8:42-7.

8. Ganji V, Zhang X, Shaikh N, Tangpricha V. Serum 25-hydroxyvitamin D concentrations are associated with prevalence of metabolic syndrome and various cardiometabolic risk factors in US children and adolescents based on assay-adjusted serum 25-hydroxyvitamin D data from NHANES 20012006. Am J Clin Nutr 2011;94:225-33.

9. Williams DM, Fraser A, Lawlor DA. Associations of vitamin D, parathyroid hormone and calcium with cardiovascular risk factors in US adolescents. Heart 2011;97:315-20.

10. Pacifico L, Anania C, Osborn JF, et al. Low 25(OH)D3 levels are associated with total adiposity, metabolic syndrome, and hypertension in Caucasian children and adolescents. Eur J Endocrinol 2011;165:603-11.

11. Roth CL, Elfers C, Kratz M, Hoofnagle AN. Vitamin d deficiency in obese children and its relationship to insulin resistance and adipokines. J Obes 2011;2011:495101.

12. Williams DM, Fraser A, Sayers A, et al. Associations of 25-hydroxyvitamin D2 and D3 with cardiovascular risk factors in childhood: cross-sectional findings from the Avon Longitudinal Study of Parents and Children. J Clin Endocrinol Metab 2012;97:1563-71.

13. Chen X, Wang Y. Tracking of blood pressure from childhood to adulthood: a systematic review and meta-regression analysis. Circulation 2008;117:3171-80. 


\section{Articles $\mid$ Kew et al.}

14. Li S, Chen W, Srinivasan SR, et al. Childhood cardiovascular risk factors and carotid vascular changes in adulthood: the Bogalusa Heart Study. JAMA 2003;290:2271-6.

15. Raitakari OT, Juonala $M$, Kähönen $M$, et al. Cardiovascular risk factors in childhood and carotid artery intima-media thickness in adulthood: the Cardiovascular Risk in Young Finns Study. JAMA 2003;290:2277-83.

16. Burguet A. Long-term outcome in children of mothers with gestational diabetes. Diabetes Metab 2010;36(6 Pt 2):682-94.

17. Boney CM, Verma A, Tucker R, Vohr BR. Metabolic syndrome in childhood: association with birth weight, maternal obesity, and gestational diabetes mellitus. Pediatrics 2005;115:e290-6.

18. Owen CG, Whincup PH, Cook DG. Breast-feeding and cardiovascular risk factors and outcomes in later life: evidence from epidemiological studies. Proc Nutr Soc 2011;70:478-84.

19. Hamilton JK, Odrobina E, Yin J, Hanley AJ, Zinman B, Retnakaran R. Maternal insulin sensitivity during pregnancy predicts infant weight gain and adiposity at 1 year of age. Obesity (Silver Spring) 2010;18: $340-6$.

20. Borgoño CA, Hamilton JK, Ye C, et al. Determinants of insulin resistance in infants at age 1 year: impact of gestational diabetes mellitus. Diabetes Care 2012;35:1795-7.
21. National Diabetes Data Group. Classification and diagnosis of diabetes mellitus and other categories of glucose intolerance. Diabetes 1979;28:1039-57.

22. Baecke JA, Burema J, Frijters JE. A short questionnaire for the measurement of habitual physical activity in epidemiological studies. Am J Clin Nutr 1982;36:936-42.

23. Retnakaran R, Qi Y, Sermer M, Connelly PW, Zinman B, Hanley AJ. Gestational diabetes and postpartum physical activity: evidence of lifestyle change 1 year after delivery. Obesity (Silver Spring) 2010;18:1323-9.

24. Matthews DR, Hosker JP, Rudenski AS, Naylor BA, Treacher DF, Turner RC. Homeostasis model assessment: insulin resistance and beta-cell function from fasting plasma glucose and insulin concentrations in man. Diabetologia 1985;28:412-9.

25. Holick MF, Binkley NC, Bischoff-Ferrari HA, et al.; Endocrine Society. Evaluation, treatment, and prevention of vitamin D deficiency: an Endocrine Society clinical practice guideline. J Clin Endocrinol Metab 2011;96:1911-30.

26. Vieth $\mathrm{R}$. Why the minimum desirable serum 25-hydroxyvitamin D level should be $75 \mathrm{nmol} / \mathrm{L}(30 \mathrm{ng} / \mathrm{ml})$. Best Pract Res Clin Endocrinol Metab 2011;25:681-91.

27. Efron B, Tibshirani R. Bootstrap methods for standard errors, confidence intervals and other measures of statistical accuracy. Stat Sci 1986;1:54-75. 